Veljan Refinements of Euler's inequalities in plane, space and $n$-space

\title{
Refinements of Euler's inequalities in plane, space and $n$-space
}

\author{
Darko Veljan \\ Department of Mathematics, University of Zagreb, Croatia \\ darko.veljan@gmail.com
}

\begin{abstract}
We improve Euler's inequality $R \geq 2 r$, where $R$ and $r$ are triangle's circumradius and inradius, respectively, and prove some consequences of it. We also show non-Euclidean version of this result. Next, we improve $3 D$ analogue of Euler's inequality for tetrahedra $R \geq 3 r$ and discuss recursive way to improve analogues of Euler's inequality for simplices. We end with some open problems, including possible CEEG (classical Euclidean elementary geometry) proof of Grace-Danielsson's inequality $d^{2} \leq(R-3 r)(R+r)$, where $d$ is the distance between the centers of the insphere and the circumsphere of a tetrahedron.
\end{abstract}

Keywords: Triangle inequalities, tetrahedron and volume inequalities, Euler's inequality in $2 D, 3 D$, and $n$-dimensional space, non-Euclidean geometry.

MSC $2010: 51 \mathrm{M} 04,51 \mathrm{M} 09,51 \mathrm{M} 16$

\section{Introduction}

In this paper we shall discuss some results about refinements of the famous Euler's inequality about the ratio of circumradius $R$ and inradius $r$ of an $n$-dimensional simplex $T$ :

$$
R \geq n r
$$

The equality is attained if and only if $T$ is a regular simplex.

DOI: https://doi.org/10.5592/CO/CCD.2020.08 
Proof. Consider the simplex $T^{\prime}$ whose vertices are centroids of faces of $T$. Then $T^{\prime}$ is clearly similar to $T$ with the similarity coefficient equal to $n$. Therefore, the circumradius $R^{\prime}$ of $T^{\prime}$ is $1 / n$ of $R$, that is, $R=n R^{\prime}$. But, $R^{\prime} \geq r$, because the smallest ball that just touch all the faces of $T$ is exactly the inscribed ball of $T$, so $R \geq n r$. It is clear that the equality is attained if and only if $T$ is a regular simplex. This completes the proof of $\left(E_{n}\right)$.

Let us recall that Euler proved the inequality $R \geq 2 r$ for triangles back in 1765 .

\section{Triangle}

The following theorem refines $\left(E_{2}\right)$, i.e. the Euler inequality $R \geq 2 r$ for triangles.

Theorem 1. For any triangle with side lengths $a, b, c$ and with circumradius $R$ and inradius $r$ we have

$$
2 a b c R \geq\left(a b c+a^{3}+b^{3}+c^{3}\right) r \geq 4 a b c r,
$$

with equality if and only if the triangle is equilateral.

Before we prove Theorem 1, let us make some remarks. Let

$$
d_{3}(x, y, z):=(x+y-z)(x-y+z)(-x+y+z) .
$$

This is a symmetric function in $x, y, z$ and hence can be expressed in terms of elementary symmetric functions $e_{1}=x+y+z, e_{2}=x y+y z+z x$, $e_{3}=x y z$. So, we have $d_{3}(x, y, z)=4 e_{1} e_{2}-e_{1}^{3}-8 e_{3}$. Schur's inequality (from 1915) tells us that $d_{3} \leq e_{3}$ (whose variables are positive reals). For an $(a, b, c)$-triangle with area $S$ and half-perimeter $s$, since $R / r=a b c / 4 S / S / s=$ $a b c / 4(s-a)(s-b)(s-c)=2 a b c / d_{3}(a, b, c) \geq 2$, it follows that $d_{3}(a, b, c) \leq a b c$, and this is a geometric proof of Schur's inequality. So, Euler's inequality $\left(E_{2}\right)$ implies Schur's inequality.

The right inequality in (1) is just the $A M-G M$ inequality for numbers $a^{3}, b^{3}$ and $c^{3}$. The left inequality in (1) is equivalent, using Heron's formula $16 S^{2}=(a+b+c) d_{3}(a, b, c)$ and formulas $R=a b c / 4 S$ and $r=2 S /(a+b+c)$ to the following inequality:

$$
(2 a b c)^{2} \geq\left(a b c+a^{3}+b^{3}+c^{3}\right) d_{3}(a, b, c) .
$$

Proof of (2) is given in [1]; one algebraic and one geometric proof; see also [5]. We provide here a short algebraic proof. (A quick proof of Heron's 
formula is to square the cosine law and the sine law $\cos C=\left(a^{2}+b^{2}-c^{2}\right) / 2 a b$, $\sin C=2 S / a b$ and use Pythagoras' rule $\cos ^{2} C+\sin ^{2} C=1$. Pythagoras' rule is equivalent to the axiom on parallels. And in fact, the cosine and the sine law and consequently Heron's formula are all equivalent to the axiom on parallels.)

Proof. Recall the cyclic sum notation: $\sum f(a, b, c):=f(a, b, c)+f(b, c, a)+$ $f(c, a, b)$, as well as the cyclic product $\prod f(a, b, c):=f(a, b, c) f(b, c, a) f(c, a, b)$. In terms of cyclic sums and products $(2)$ can be written as

$$
4 \prod a^{2} \geq\left(\prod a+\sum a^{3}\right) d_{3}(a, b, c) .
$$

It is easy to check by expansion that in terms of cyclic sums we have the following sum of squares:

$d_{3}(a, b, c)^{2}-d_{3}\left(a^{2}, b^{2}, c^{2}\right)=\sum\left(a\left(b^{2}-b c+c^{2}-a^{2}\right)\right)^{2}+\left(\sum a\left(b^{2}-b c+c^{2}-a^{2}\right)\right)^{2} \geq 0$.

On the other hand (as it is again easy to check):

$$
d_{3}(a, b, c)^{2}-d_{3}\left(a^{2}, b^{2}, c^{2}\right)=2(2 a b c)^{2}-2\left(a b c+a^{3}+b^{3}+c^{3}\right) d_{3}(a, b, c) .
$$

From the last two identities and inequalities we obtain (3), and hence (2).

If we express everything in (2) in terms of elementary symmetric functions $e_{1}, e_{2}, e_{3}$ in variables $a, b, c$, we obtain the following symmetric inequality in side lengths for any triangle (note, $\prod a+\sum a^{3}=e_{1}^{3}-3 e_{1} e_{2}+4 e_{3}$ ):

$$
e_{1}^{6}+12 e_{1}^{3} e_{3}+12 e_{1}^{2} e_{2}^{2}+36 e_{3}^{2} \geq 7 e_{1}^{4} e_{2}+40 e_{1} e_{2} e_{3} .
$$

Again, this inequality is tight in the sense that it becomes equality if and only if the triangle is equilateral. We shall apply our refinement of Euler's inequality to upper bound the area of a triangle.

Theorem 2. The area $S$ of a triangle with side lengths $a, b, c$ can tightly be bounded from above as

$$
4 S^{2} \leq \frac{(a+b+c) a^{2} b^{2} c^{2}}{a b c+a^{3}+b^{3}+c^{3}} \leq \frac{(a+b+c)^{4}}{2^{2} 3^{3}} .
$$

Proof. The left hand side of (5) is equivalent to (1). Namely, (1) can be stated as

$$
\frac{R}{r}=\frac{(a+b+c) a b c}{8 S^{2}} \geq \frac{a b c+a^{3}+b^{3}+c^{3}}{2 a b c},
$$

and this inequality is obviously equivalent to the left inequality in (5). The right inequality in (5) follows from the AM-GM inequality $a b c \leq\left(\frac{a+b+c}{3}\right)^{3}$ and the inequality $\frac{a b c}{a b c+a^{3}+b^{3}+c^{3}} \leq 1 / 4$, and this is equivalent to the AM-GM inequality $a b c \leq \frac{a^{3}+b^{3}+c^{3}}{3}$. 
Note that (5) is an improvement of the standard isoperimetric inequality for triangles

$$
S \leq \frac{\sqrt{3}(a+b+c)^{2}}{36} .
$$

This is also explained in [1] and [5]. The left inequality in (5) improves the inequality $S \leq \frac{3(a b c)^{2 / 3}}{4}$, which, in turn, improves the standard isoperimetric inequality for triangles, as proved by Theorem 4.1. in [11]. So, we have the following chain of bounds for the area $S$ of an $(a, b, c)$-triangle:

$$
4 S^{2} \leq \frac{(a+b+c) a^{2} b^{2} c^{2}}{a b c+a^{3}+b^{3}+c^{3}} \leq \frac{3(a b c)^{4 / 3}}{4} \leq \frac{(a+b+c)^{4}}{2^{2} 3^{3}} .
$$

\section{Noneuclidian triangles}

The following Lemma from [2] allows us to convert inequalities for Euclidean into spherical and hyperbolic triangles.

Lemma 3. If $f(a, b, c) \geq 0$ is an inequality which holds for all Euclidean triangles with side lengths $a, b, c$, then $f(s(a), s(b), s(c)) \geq 0$ for all spherical or hyperbolic triangles with side lengths $a, b, c$, where $s(x)=x / 2$ in Euclidean geometry, $s(x)=\sin (x / 2)$ in spherical geometry and $s(x)=\sinh (x / 2)$ in hyperbolic geometry.

Using this Lemma, the inequality (2) or (4) can easily be converted into corresponding spherical or hyperbolic analogues. This is elaborated in more details in [1] and [5]. For instance, in hyperbolic geometry, the inequality (4) holds with symmetric functions in variables $\sinh (a / 2)$ etc.

\section{Tetrahedron}

Now we go to the 3 -dimensional $(3 D)$ space. Let $T=A B C D$ be a tetrahedron (3-simplex) with edge lengths $a, b, c, a^{\prime}, b^{\prime}, c^{\prime},(a, b, c$ forming the triangle $A B C$, a against $a^{\prime}$ etc. $)$, volume $V=\operatorname{vol}(A B C D)$, surface area $S(=$ $\left.S_{A}+\ldots+S_{D}\right)$ and $R$ and $r$ the circumradius and inradius of $T$, respectively. It is well known that the numbers $a a^{\prime}, b b^{\prime}$ and $c c^{\prime}$ are side lengths of the triangle called Crelle's triangle of $T$. Recall, Crelle's triangle $A^{*} B^{*} C^{*}$ of $T$ against $D$ is the image of the triangle $A B C$ under the inversion $I$ with the center $D$ and radius $\rho$ whose square is equal $\rho^{2}=D A \cdot D B \cdot D C=a^{\prime} b^{\prime} c^{\prime}$. $A^{*}=I(A)$, etc. (Recall, the inversion $I$ maps a point $X \neq D$ to the point $X^{*}$ on the ray $D X$, such that the product of distances is $D X \cdot D X^{*}=\rho^{2}$; point $D$ is mapped to the infinity.) There are three more Crelle's triangles. 
The area Crelle of the Crelle triangle is given by the Crelle formula: Crelle $=6 R V$ (see e.g. [3]).

Proof of Crelle's formula. The distance $h$ of $D$ to the plane $A^{*} B^{*} C^{*}$ is equal to $\rho^{2} / 2 R$, because the circumsphere of $T$ is mapped by $I$ to the plane $A^{*} B^{*} C^{*}$. The ratio of volumes $\operatorname{vol}\left(D A^{*} B^{*} C^{*}\right): \operatorname{vol}(D A B C)$ is equal to the ratio of products of lengths of lateral edges, that is

$$
\operatorname{vol}\left(D A^{*} B^{*} C^{*}\right): \operatorname{vol}(D A B C)=\frac{D A^{*} \cdot D B^{*} \cdot D C^{*}}{D A \cdot D B \cdot D C} .
$$

Namely, if $h_{C}$ and $h_{C}^{*}$ are distances from $C$ and $C^{*}$ to the plane $D A B$ (or $D A^{*} B^{*}$ ) and $S_{C}, S_{C}^{*}$ the areas of triangles $D A B$ and $D A^{*} B^{*}$, respectively, then $h_{C}: h_{C}^{*}=D C: D C^{*}$ and $S_{C}: S_{C}^{*}=(D A \cdot D B):\left(D A^{*} \cdot D B^{*}\right)$ and since $V=\operatorname{vol}(D A B C)=S_{C} h_{C} / 3, \operatorname{vol}\left(D A^{*} B^{*} C^{*}\right)=S_{C}^{*} h_{C}^{*} / 3$, it follows that

$$
\frac{\operatorname{vol}\left(D A^{*} B^{*} C^{*}\right)}{\operatorname{vol}(D A B C)}=\frac{h_{C}^{*} S_{C}^{*}}{h_{C} S_{C}}=\frac{D A^{*} \cdot D B^{*} \cdot D C^{*}}{D A \cdot D B \cdot D C} .
$$

So,

$\operatorname{vol}\left(D A^{*} B^{*} C^{*}\right)=V \cdot \frac{D A^{*}}{D A} \cdot \frac{D B^{*}}{D B} \cdot \frac{D C^{*}}{D C}=V \cdot\left(\frac{\rho}{D A}\right)^{2} \cdot\left(\frac{\rho}{D B}\right)^{2} \cdot\left(\frac{\rho}{D C}\right)^{2}=V \rho^{2}$.

On the other hand, $\operatorname{vol}\left(D A^{*} B^{*} C^{*}\right)=$ Crelle $\cdot \frac{h}{3}$, so $V \rho^{2}=$ Crelle $\cdot \frac{h}{3}=$ $\left(\frac{\text { Crelle }}{3}\right) \rho^{2}$, and hence Crelle $=6 R V$. By symmetry, the other three Crelle's triangles have the same area.

By using Crelle's formula, the standard isoperimetric inequality for tetrahedra (see [3]) can be derived, as we shall prove later in the text

$$
2^{3} 3^{4} V^{2} \leq \sqrt{3} S^{3}
$$

The following inequality (see [4]) is also crucial for the rest of our argument:

$$
2^{3} 3^{2} V^{2} \leq d_{3}\left(a a^{\prime}, b b^{\prime}, c c^{\prime}\right) .
$$

Proof of (8). By Heron's formula for Crelle's triangle and by Crelle's formula we have $16 C r e l l e^{2}=\left(a a^{\prime}+b b^{\prime}+c c^{\prime}\right) d_{3}\left(a a^{\prime}, b b^{\prime}, c c^{\prime}\right)=16(6 R V)^{2}$. So, (8) is equivalent to

$$
a a^{\prime}+b b^{\prime}+c c^{\prime} \leq 8 R^{2}
$$

(with equality iff $T$ is equifacial). 
To prove (9), recall Lagrange's center mass theorem, which says that if $A_{1}, \ldots, A_{n}$ is any set of points in space and $G$ their centroid (center of mass), then $\sum \overrightarrow{G A_{i}}=\mathbf{0}$. For any point $P$, the moment of inertia $I_{P}$ is defined as $I_{P}:=\sum P A_{i}^{2}$. Then

$$
I_{P}=\sum\left(\overrightarrow{P G}+\overrightarrow{G A_{i}}\right)^{2}=\sum G A_{i}^{2}+n P G^{2}+2 \overrightarrow{P G} \cdot \sum \overrightarrow{G A_{i}}=I_{G}+n P G^{2} .
$$

By adding these equalities for $P=A_{1}, \ldots, A_{n}$, we get

$$
2 \sum_{i<j} A_{i} A_{j}^{2}=\sum I_{A_{i}}=n I_{G}+n \sum G A_{i}^{2}=2 n I_{G} .
$$

From the last two equalities we obtain

$$
n I_{P}=\sum_{i<j} A_{i} A_{j}^{2}+n^{2} P G^{2} .
$$

For $n=4$ and $A_{1}, \ldots, A_{4}=A, B, C, D$ the vertices of $T$, this implies that for any point $P$ in space we have

$$
4 \sum P A^{2}=\sum a^{2}+16 P G^{2} .
$$

If $P=O$, the circumcenter of $T$, then the last equality implies

$$
16 R^{2}=\sum a^{2}+16 O G^{2}
$$

Since $a^{2}+a^{\prime 2} \geq 2 a a^{\prime}$, etc., (9) follows since $O G^{2} \geq 0$. This proves the inequality (8). Equality holds if and only if $a=a^{\prime}, b=b^{\prime}$ and $c=c^{\prime}$, and $G=O$. On the other hand, if $T$ is equifacial, then $G=O$, because if $P$ is any of the vertices of $T$ (recall, $a=a^{\prime}$ etc.), from above equality we get by symmetry that $G$ has the same distance from all four vertices of $T$, so $G=O$.

We shall prove (see [1]) the following refinement, or rather improvement of Euler's inequality $\left(E_{3}\right)$, i.e. $R \geq 3 r$.

\section{Theorem 4 .}

$$
\left(\frac{R}{r}\right)^{2} \geq \frac{3\left(a a^{\prime}+b b^{\prime}+c c^{\prime}\right)}{d_{3}^{1 / 3}} \geq 9 .
$$

Here, $d_{3}=d_{3}\left(a a^{\prime}, b b^{\prime}, c c^{\prime}\right)$. Equality on the left hand side of (10) becomes equality if and only if the tetrahedron $T$ is equifacial, i.e. $a=a^{\prime}, b=b^{\prime}$ and $c=c^{\prime}$. The right inequality becomes equality if and only if $a a^{\prime}=b b^{\prime}=c c^{\prime}$ (i.e. if and only if the Crelle triangle is equilateral). 
Proof. Start with Crelle's formula and the equality $3 V=r S$. We have $(C$ is the area of Crelle's triangle)

$$
\left(\frac{R}{r}\right)^{2}=\frac{C^{2} S^{2}}{2^{2} 3^{4} V^{4}}=\frac{16 C^{2}}{2^{6} 3^{4}} \cdot\left(\frac{S^{3}}{V^{2}}\right)^{2 / 3} \cdot \frac{1}{V^{8 / 3}} .
$$

Heron's formula for the Crelle triangle yields

$$
16 C^{2}=\left(a a^{\prime}+b b^{\prime}+c c^{\prime}\right) d_{3}\left(a a^{\prime}, b b^{\prime}, c c^{\prime}\right) .
$$

The isoperimetric inequality (7) tells us that $\frac{S^{3}}{V^{2}} \geq \frac{2^{3} 3^{4}}{\sqrt{3}}$. The inequality (8) says

$$
\frac{1}{V^{8 / 3}} \geq \frac{2^{4} \cdot 3^{8 / 3}}{d_{3}\left(a a^{\prime}, b b^{\prime}, c c^{\prime}\right)^{4 / 3}} .
$$

Inserting these estimates to (11) yields the left inequality in (10). The right inequality in (10) follows from the Schur inequality and the AM-GM inequality for the numbers $a a^{\prime}, b b^{\prime}, c c^{\prime}$. Namely,

$$
d_{3}\left(a a^{\prime}, b b^{\prime}, c c^{\prime}\right) \leq\left(a a^{\prime}\right)\left(b b^{\prime}\right)\left(c c^{\prime}\right) \leq\left(\frac{a a^{\prime}+b b^{\prime}+c c^{\prime}}{3}\right)^{3}
$$

It is clear that the left inequality in (10) becomes an equality if and only if both (7) and (8) are equalities, i.e. if and only if the tetrahedron is equifacial.

\section{Simplex}

Finally, we go to the $n$-dimensional space. With obvious notations we have the following standard isoperimetric inequality for an $n$-simplex $T=A_{0} \ldots A_{n}$ with basically the same proof as for tetrahedra:

$$
\left(n ! S^{n}\right)^{2} \geq n^{3 n}(n+1)^{n+1} V^{2(n-1)} .
$$

In fact, even a stronger upper bound for $V$ holds in terms of the product of surface areas $S_{i}$ of $T$ (see [3], [6], [7]):

$$
V^{2(n-1)} \leq(n !)^{2} \frac{(n+1)^{n-1}}{n^{3 n}}\left(S_{0} \times S_{1} \times \cdots \times S_{n}\right)^{2 n /(n+1)} .
$$

For reader's covenients, and since (12) and (13) are not very well known, we provide a proof of them bellow in the text (for $n=3$ ). 
Next, as we know the crux of the proof of (8) is the inequality $16 R^{2} \geq$ $\sum a^{2}$, where $\sum a^{2}$ is the sum of squared edge lengths of $T$. The same proof works for an $n$-simplex $T$ :

$$
(n+1)^{2} R^{2} \geq \sum a^{2} .
$$

Define Crelle's (n-1)-simplex $C_{n-1}^{(0)}$ of the simplex $T=A_{0} A_{1} \ldots A_{n}$ against the vertex $A_{0}$ of the simplex $T$ as the image $A_{1}^{*} A_{2}^{*} \ldots A_{n}^{*}=I_{0}\left(A_{1} \ldots A_{n}\right)$, $A_{i}^{*}=I_{0}\left(A_{i}\right), i=1, \ldots, n$, where $I_{0}=I_{0}\left(A_{0}, \rho_{0}\right), \rho_{0}^{2}=\prod_{i=1}^{n} A_{0} A_{i}$, is the inversion with the center $A_{0}$ and the radius of inversion $\rho_{0}$.

The ratio of volumes of pyramides $A_{0} A_{1}^{*} \ldots A_{n}^{*}$ and $A_{0} A_{1} \ldots A_{n}$ is equal to the ratio of products of side lengths of lateral edges $A_{0} A_{1}^{*} \times \cdots \times A_{0} A_{n}^{*}$ and $A_{0} A_{1} \times \cdots \times A_{0} A_{n}$. Little calculation yields that the volume is equal to:

$$
\operatorname{vol}_{n-1}\left(C_{n-1}^{(0)}\right)=2 n R V \rho_{0}^{2(n-3)} .
$$

For $n=3$, formula (15) reduces to Crelle's formula $C=6 R V$. In order to symmetrize everything, consider $C_{n-1}$, the product of all $\operatorname{vol}_{n-1}\left(C_{n-1}^{(i)}\right)$, $i=0,1, \ldots, n$, where $C_{n-1}^{(i)}$ is Crelle's $(n-1)$-simplex of $T$ against the vertex $A_{i}$. Note that for $n=2, C_{1}=a b c=4 R S$, and (1) or (5) can be applied. Also, $C_{2}=(6 R V)^{4}$ and $C_{3}=(8 R V)^{5} \prod a^{2}$.

Now we can recursively improve Euler's inequality $\left(E_{n}\right), n>3$, as follows. Start with $n=4$ and apply (8) to all five Crelle's tetrahedra in $C_{3}$. Then use (14). This gives an upper bound for the volume $V$ of a 4 -simplex $T$ in terms of its edge lengths. Then use again (14) and $r S=4 V,(13),(14)$ to estimate $R / r$. Then continue that way to higher dimensions.

The obtained intrinsic lower bound for $R / r$ is certainly better than the ambiental Euler's bound $n$. We shall not go into details here, but the interested reader can check that the procedure works on a simple example with 4-simplex, say, origin and four unit vertices on coordinate axes.

\section{Isoperimetric and some other inequalities}

We shall now prove (12) and (13) for $n=3$, but the same proof by "mutatis mutandis" works for all $n$. Let us prove (7), i.e.

$$
\frac{S^{3}}{V^{2}} \geq 2^{3} 3^{3} \sqrt{3}
$$

with equality if and only if $T$ is equifacial. 
Proof. Let $T=A B C D$ be a tetrahedron and $S_{A}, \ldots, S_{D}$ be face areas of $T$ ( $S_{A}$ opposite to $A$ etc.); in the sum they give $S$. Let $\mathbf{n}_{A}$ be the unit outward normal vector to the face with area $S_{A}$ etc. Then the vector sum $\sum S_{A} \mathbf{n}_{A}=0$ (because vector on the left is perpendicular to all vectors including itself, so it has to be zero). Let $W_{A}$ be the volume of the tetrahedron $T_{A}$ spanned by $\mathbf{n}_{B}$, $\mathbf{n}_{C}, \mathbf{n}_{D}$ and similarly $W_{B}$ and so on. Then we have $W_{A} S_{B} S_{C} S_{D}=3 V^{2} / 4$, as well as the other three such formulas (this is called the sine law for tetrahedra, see [6]). Indeed, by multiplying the above null-sum of vectors by $\times S_{A} \mathbf{n}_{A}$ (cross product) and then by $S_{B} \mathbf{n}_{B}$ (scalar product), and taking into account that $W_{A}=\left(\left(\mathbf{n}_{B} \times \mathbf{n}_{C}\right) \mathbf{n}_{D}\right) / 6$ and so on, the claim above follows easily. Next, we need the inequality $27 \mathrm{~V} \leq 8 \sqrt{3} \cdot R^{3}$. To prove this, let $d$ be the distance from the circumcenter of $T$ to the plane $B C D$ and $R_{A}$ the circumradius of the triangle $B C D$. Then $R_{A}^{2}=R^{2}-d^{2}$ and $V \leq S_{A}(R+d) / 3$, because the altitude to $B C D$ is at most $R+d$. Recall that the area $K$ of any triangle with side lengths $a, b, c$ satisfies the inequality $K \leq \sqrt{3}(a b c)^{2 / 3} / 4$, or equivalently, $K \leq 3 \sqrt{3} \cdot R^{2} / 4$. Hence,

$$
S_{A} \leq 3 \sqrt{3} \cdot \frac{R_{A}^{2}}{4}=\frac{3 \sqrt{3}}{4}\left(R^{2}-d^{2}\right) .
$$

Therefore,

$$
V \leq \frac{S_{A}}{3}(R+d) \leq \frac{\sqrt{3}}{4}\left(R^{2}-d^{2}\right)(R+d)
$$

The AM-GM inequality yields

$$
2\left(R^{2}-d^{2}\right)(R+d)=(2 R-2 d)(R+d)(R+d) \leq\left(\frac{4 R}{3}\right)^{3} .
$$

Thus, finally, $V \leq 8 \sqrt{3} \cdot R^{3} / 27$. Now, all four tetrahedra $T_{A}, \ldots, T_{D}$ with volumes $W_{A}, \ldots, W_{D}$, respectively, do not intersect in their interiors and their union $E$ is a tetrahedron inscribed in the unit sphere. By the above discussion, the volume $W$ of $E$ is at most $8 \sqrt{3} / 27$. Hence, by the AM-GM we have

$$
\prod W_{A} \leq\left(\sum \frac{W_{A}}{4}\right)^{4}=\left(\frac{W}{4}\right)^{4} \leq\left(\frac{8 \sqrt{3}}{27 \cdot 4}\right)^{4}=\frac{2^{4}}{3^{10}}
$$

Finally, by multiplying all four sine-law formulas, by using above results and the AM-GM we get

$$
V^{4}=\frac{16}{9} \cdot\left(\prod S_{A}\right)^{3 / 2}\left(\prod W_{A}\right)^{1 / 2} \leq \frac{16}{9} \cdot\left(\sum \frac{S_{A}}{4}\right)^{6} \cdot \frac{2^{2}}{3^{5}}=\frac{S^{6}}{2^{6} 3^{7}}
$$

By taking the square root (16) follows. Equality is clear from the proof. 
The same proof (with obvious changes) works for any simplex, so we have just proved (12) and (13). Some consequences of the above proof and (13) are the inequalities (see also [6], [7]):

$$
(n ! V)^{2} n^{n} \leq(n+1)^{n+1} R^{2 n}, \quad(n ! V)^{2} 2^{n} \leq(n+1)\left(\prod a^{2}\right)^{2 /(n+1)}
$$

with equalities if and only if the simplex is regular.

The right inequality in (17) was first conjectured by this author back in 1969, when as an undergraduate student proposed it as an open problem in Elemente der Mathematik, and then proved three years later by G. Korchmáros, see [8] and [9]. In 1981, J. Zh. Zhang and L. Yang gave it a different aspect, [10]. Further development can be traced in [6] and [7].

\section{$7 \quad$ Some open problems}

We end with some open problems. In [11] we proved non- Euclidean versions of some classical triangle inequalities. The problems and proposals for new research projects are the following.

What are non-Euclidean versions of some of the 3-dimensional or $n$ dimensional inequalities described in this paper and some other ("classical") inequalities for tetrahedra and simplices?

For example, we can apply the inequalities (2) or (4) to Crelle's triangle of a (Euclidean) tetrahedron and thus obtain the corresponding inequality for tetrahedra, symmetric in products of opposite edges. Then by Lemma 3 it can be applied to hyperbolic (and spherical) tetrahedra. What is $3 D$ analogue of Lemma 3? And in higher dimensions?

Next, what is the hyperbolic version of (8) and of our main inequality (10), when hyperbolic tetrahedron has circumsphere?

Note that for a hyperbolic tetrahedron we can form the corresponding Crelle's triangle with side lengths as products of sinh of (halfs of) opposite dihedral angles, and similarly in the spherical case. What is the hyperbolic $3 D$-analogue of Crelle's formula? And more generally of formula (15) for hyperbolic simplices?

What are hyperbolic (and spherical) versions of (12), (13) and (17)?

It is well known that computing volumes is a hard task even in Euclidean geometry and needless to say in hyperbolic geometry. Even for a simplex, computing Cayley-Menger determinant is a hard task. Therefore, having on disposal various upper (and lower) bounds for volume of a tetrahedron could be important in many applications in physics, astrophysics, IT technology, crystallography and other disciplines when using triangulations in order to 
Veljan Refinements of Euler's inequalities in plane, space and $n$-space

estimate the volume of a body. For instance, one could imagine that in future we could want to estimate the volume in deep space determined by four (or more) stars, knowing their mutual distances. Then this volume should be estimated by an inequality for a hyperbolic tetrahedron in the (supposedly hyperbolic) universe and sum all of them.

The last problem is about Grace-Danielsson's inequality (see (18)). It was proposed in 1949, but without clear proof by Danielsson and claimed earlier by Grace. If $d=O I$ is the distance between the circumcenter $O$ and the incenter $I$ of the tetrahedron $T$, then

$$
d^{2} \leq(R-3 r)(R+r) .
$$

If $T$ is equifacial then $O=I$ and since $R \geq 3 r,(18)$ holds in this case. Can our results provide a proof in general case? Is there a short elegant proof of (18) in the sense of CEEG (classical Euclidean elementary geometry)? Recall, Euler proved in 1767 that for a triangle $d^{2}=(R-2 r) R$. A short proof is by inversion w.r.t. the incircle of a triangle: the image of the circumcircle has radius $r^{*}=r / 2=R r^{2} /\left(R^{2}-d^{2}\right)$. At the moment it's not clear whether a similar method works for tetrahedra. The inequality (18) was proved via computer aided argument and by quantum information theory in 2015 and 2018 (see lit. in [5]). So, the interest for finding a proof of (18) even among computer scientists shows its importance. In higher dimensions there are not even any documented hypothesis analogous to Euler's formula or (18) inequality. Perhaps some arguments involving Crelle's triangle or Crelle's simplices could be helpful in higher dimensions and allow us to use recursive method as we did with Euler's inequality. At the moment it is not known how to approach geometrically to this elusive inequality. In any case, it is a good project for further research.

\section{References}

[1] D. Veljan, Improved Euler's inequality in plane and space, Journal of Geometry, to appear

[2] R. Guo, E. Black, C. Smith, Strengthened Euler's inequality in spherical and hyperbolic geometry, arXiv: 1704.053373v.[math MG] 17 April 2017.

[3] B. Pavković, D. Veljan, Elementarna matematika II (in Croatian), Tehnička knjiga-Goldenmarketing, Zagreb, 1994. 
Veljan Refinements of Euler's inequalities in plane, space and $n$-space

[4] M. Mazur, An inequality for the volume of a tetrahedron, Amer. Math. Monthly 125 (2018) 273-275.

[5] D. Veljan, Two inequalities: a geometric and a combinatorial, Proc. 2nd Croatian Combinatorial Days, Zagreb, September 27-28, 2018 (Eds. T. Došlić and I. Martinjak), 183-194.

[6] D. Veljan, The sine theorem and inequalities for volumes of simplices and determinants, Lin. Alg. Appl. 219 (1996) 79-91.

[7] V. Volenec, D. Veljan and J. Pečarić, Inequalities for volumes of simplices in terms of their faces, Geom. Dedicata 37 (1998) 57-62.

[8] D. Veljan and G. Korchmáros, Aufgabe 629 A, Elemente der Math. 27 (1972) 14-15.

[9] G. Korchmáros, Una limitazzione per il volume di un simplesso ndimensionale avente spigoli di date lunghezze, Atti Acad. Lincei, Rend. (8) 56 (1974), 876-879.

[10] J. Zh. Zhang and L. Yang, A class of geometric inequalities concerning the mass-point system (in Chinese), J. China Univ. Sci. Tech. 11 (1981) $1-8$.

[11] D. Svrtan and D. Veljan, Non Euclidean versions of some classical triangle inequalities, Forum Geom. 12 (2012) 197-209. 